\title{
Representaciones docentes de la Educación Basada en Competencias. Un estudio de caso
}

\section{Teaching Representations of Competency-Based Education. A Case Study}

\section{Patricia Covarrubias-Papahiu}

Universidad Nacional Autónoma de México, Ciudad de México, México.

Doctora en Pedagogía por la Facultad de Filosofía y Letras de la UNAM. Profesora Titular C de la carrera de Psicología, Facultad de Estudios Superiores (FES) Iztacala, UNAM.

Recibido: 02-02-16

Aprobado: 20-04-16

\section{*Correspondencia}

Email: papahiu@unam.mx
Citar como:

Covarrubias-Papahiu, P. (2016).

Representaciones docentes de la Educación Basada en Competencias. Un estudio de caso. Propósitos y Representaciones, 4(2), 73-132. Doi:

http://dx.doi.org/10.20511/pyr2016.v4n2.120

\footnotetext{
(C) Universidad San Ignacio de Loyola, Vicerrectorado de Investigación y Desarrollo, 2016. (c) BY.NC-ND Este artículo se distribuye bajo licencia CC BY-NC-ND 4.0 Internacional (http://creativecommons.org/licenses/by-nc-nd/4.0/).
} 


\section{Resumen}

El propósito de la investigación fue conocer cómo representan el enfoque de la Educación Basada en Competencias (EBC) profesores que participan de la formación profesional de psicólogos, y los retos e implicaciones que desde su perspectiva tiene incorporarlo en la práctica del aula. Para ello se indagaron el tipo de representaciones y sentidos que le otorgan al enfoque para su aceptación o rechazo, si emplean este en su práctica docente, y el tipo de competencias que les interesa promover en sus estudiantes para su ejercicio profesional. Como estudio de caso, en el que colaboraron profesores de la carrera de Psicología que se imparte en uno de los campos universitarios de la UNAM, se utilizó la metodología de corte cualitativa, con enfoque descriptivo e interpretativo, teniendo como instrumento de recolección de datos la entrevista semiestructurada. Los resultados más distintivos muestran que en la heterogeneidad de representaciones develadas entre los docentes entrevistados, la balanza se inclina hacia los docentes que no aprueban y/o no emplean el enfoque basado en competencias. Entre los argumentos más consistentes de estos para su rechazo están el desconocimiento del enfoque o dificultad para llevarlo a la práctica, y/o su vinculación con las políticas de corte neoliberal, funcionalista y empresarial. Sin embargo, quienes sí lo aceptan y aplican valoran el desarrollo de actitudes que contempla el enfoque, principalmente los que tienen una visión humanística para la formación de psicólogos, y otros aprecian que promueve las capacidades para un desempeño profesional eficiente.

Palabras claves: Educación basada en competencias, representaciones sociales, formación profesional, psicólogos, profesores universitarios.

\section{Summary}

The aim of this research was to know how the Competency-Based Education (CBE) approach is represented by professors who are part of the professional education of psychologists, and the challenges and implications of, in their 
opinion, incorporating it in the classroom practice. Therefore, a research was conducted to know the type of representations and meanings that they give to the approach for its approval or rejection, whether they apply it in their teaching practice, and the kind of competencies that they want to promote among their students for their professional practice. This case study that counts with the collaboration of professors from the Psychology Specialty delivered in one of the university campuses of the UNAM University, used a qualitative methodology with a descriptive and interpretative approach that used semi-structured interviews as a data collection tool. The most distinctive results demonstrate that, concerning the heterogeneous nature of the disclosed representations among the interviewed professors, the balance falls in favor of the teachers that do not approve and/or use the competencybased approach. Their most consistent arguments to reject it included the unfamiliarity with the approach, or the difficulty to put it into practice, and/or its connection with neo-liberal, functionalist and business policies. However, the professors that accept and apply it, value the development of attitudes considered by the approach, especially those with a humanistic view towards the education of psychologists; while others appreciate how it promotes the necessary abilities for an efficient professional performance.

Keywords: Competency-based education, social representations, professional education, psychologists, professors. 


\section{Introducción}

El tema de las competencias ha ocupado un lugar preponderante en la educación de nuestro país desde finales del siglo pasado y ha sido motivo de debate y controversias entre los especialistas del currículo y los actores del mismo.

Tomando en consideración la importancia que ha cobrado la enseñanza por competencias en nuestro país y las discusiones sobre su adecuación al contexto mexicano, el trabajo que presentamos pretende aportar información que contribuya al entendimiento de las problemáticas, controversias y retos que para profesores universitarios representa el enfoque por competencias en su práctica educativa. ${ }^{1}$

Como antecedentes del caso se encuentran las políticas educativas de la última década orientadas a elevar la calidad educativa que impulsaron reformas curriculares en todos los niveles educativos, sustituyendo los modelos pedagógicos tradicionales centrados en la enseñanza por modelos más centrados en el aprendizaje. Esto promovió el aumento de investigaciones que dieran cuenta, desde lo vivido y desde la mirada de sus actores, del significado que estas reformas e innovaciones curriculares y pedagógicas tuvieron para ellos, con lo cual se empezó a tener un material empírico y básico para su valoración.

En las investigaciones realizadas es frecuente hallar que existe una serie de inconsistencias, resistencias y estrategias que los docentes adoptan para enfrentarse a los cambios e innovaciones curriculares, y en las que mucho se ha insistido en que estas, por sí mismas, no habilitan a los profesores en los cambios necesarios y, por lo tanto, existe la necesidad de indagar por qué no llegan a alcanzar el éxito previsto (Covarrubias \& Casarini, 2013).

1 Los resultados forman parte de la línea de investigación "Procesos curriculares y prácticas pedagógicas en educación superior", del proyecto de investigación Evaluación y Planeación Educativa de la FES Iztacala-UNAM. Avances de estos resultados fueron presentados en el IV Congreso ALFEPSI: Identidad, Innovación y Compromiso Social, en la Ciudad de Santa Marta, Colombia, octubre 2015. 
En el caso particular del enfoque de la Educación Basada en Competencias (EBC), su discusión constante por especialistas e investigadores parte de, por lo menos, dos temas recurrentes. Por un lado se encuentra el problema que representa la polisemia del término competencia, pues es tal la multiplicidad de acepciones que se le asignan que se prefiere hablar de la noción de competencias en lugar de su concepto, en tanto se está lejos de disponer de una definición precisa, operativa y mínimamente consensuada del término (Winterton, Delamare y Stringfelow, 2006; Planas-Coll, 2013); y por otro lado están las implicaciones teórico-prácticas que conlleva el enfoque por competencias tanto en los procesos curriculares como en los didácticos (Díaz Barriga, 2013).

Aun con estas limitaciones, la EBC se ha adoptado en nuestro país de manera extendida. En la educación básica y media superior, la incorporación del enfoque por competencias ha sido el resultado de las reformas curriculares y educativas que se han implementado en la última década. En lo que toca a las Instituciones de Educación Superior (IES), aun cuando fueron estas las que iniciaron su utilización en sus planes de estudio, se ha promovido a través de la implementación de modelos educativos y académicos propios, y con base en la autonomía de la que gozan, deciden su utilización, el momento y el enfoque de competencias a utilizar (Díaz Barriga, F. et al., 2013).

No obstante esta incorporación curricular del enfoque para la formación de diversos profesionistas, como médicos, abogados, contadores, entre otros, la enseñanza por competencias, como novedad o como innovación educativa, aún es motivo de controversia hoy en día (Díaz Barriga, 2013). No existe consenso ni claridad sobre cómo debe llevarse a cabo un programa basado en competencias; los nuevos currículos universitarios que se desarrollan en el país con este enfoque comprenden diferentes formas de interpretarlo y diversas formas de enseñanza y aprendizaje (Moreno, 2010). De aquí que, a pesar de la aceptación que a nivel curricular ha alcanzado, para los investigadores en el campo de la didáctica falta indagar lo que en la práctica se entiende e implementa como educación basada en competencias. 
En el caso particular de la psicología, aunque es indudable su legitimidad en el mundo contemporáneo por sus aportaciones al conocimiento y el tratamiento de una variedad de problemas a nivel individual y grupal en diversos ámbitos de la actuación humana, cuando se analiza como profesión existe una intensa discusión sobre la efectividad de su ejercicio profesional y su relevancia social. Entre los factores que se señalan están la excesiva teorización y transmisión de sus contenidos disciplinarios y la poca preparación en habilidades o competencias, que se traduce en un desfase entre la formación profesional del psicólogo y las necesidades ocupacionales y sociales de nuestra sociedad (Preciado \& Rojas, 1989; Almeida, Guarneros, Limón \& Román, 1989; Castaño, 1989; Lara, 1989; Herrera, 1993; Harrsch, 1998; Castañeda, 1999; Covarrubias, 2003; Covarrubias \& Camarena, 2010; Zanatta \& Yurén, 2012). Y efectivamente, cuando la formación profesional no alcanza a articular el saber científico y las demandas sociales, esta misma se pone en entredicho en tanto se considera alejada o descontextualizada de lo que significa la vida profesional.

En tal sentido, nos planteamos como interrogante inicial si el enfoque por competencias representaría una alternativa para resolver los problemas que se confieren a la enseñanza de la psicología, en tanto, e independientemente de la perspectiva o escuela de pensamiento con el que se aborde, contiene dos características que se encuentran implícitas en cualquier definición de competencia: por un lado se centra en el desempeño y, por el otro, recupera condiciones concretas de la situación en que dicho desempeño es relevante (Victorino \& Medina, 2007). No obstante, y como ya se ha documentado suficientemente, el profesor es el primer mediador entre las prescripciones curriculares o innovaciones educativas y la práctica pedagógica (Young, 1981; Gimeno y Pérez, 1989; Gimeno, 1995), por lo que destaca la importancia de prestar atención a las formas en que los docentes representan, conciben o interpelan el enfoque por competencias para su adecuación a las necesidades de formación profesional del psicólogo. En este sentido, la investigación que presentamos tuvo como propósito indagar las representaciones y sentidos que profesores de psicología de un campo universitario de la UNAM le otorgan 
al enfoque para su aceptación o rechazo, si emplean este en su práctica docente y, en su caso, el tipo de competencias que les interesa promover en sus estudiantes.

Desde aquí, las interrogantes que guiaron nuestra investigación fueron: ¿Cómo representan o conciben la Educación Basada en Competencias (EBC) profesores que participan en la formación profesional de psicólogos en un espacio universitario en particular? ¿Qué significados, representaciones o valoraciones le asignan al enfoque para su aceptación o rechazo? ¿Se tiene preferencia por este enfoque según el área de la psicología en la que enseñan los profesores? y, en su caso, ¿qué competencias les interesa promover?

Optamos por la metodología cualitativa en su modalidad de estudio de caso como la mejor forma de indagación para encontrar respuestas a nuestras interrogantes, en tanto nuestro interés se centró en comprender lo que pasa en un contexto y en una práctica curricular en particular (Stake, 1999), como es el caso de la carrera de Psicología que se imparte en uno de los campos multidisciplinarios de la UNAM; carrera que se ha caracterizado por la diversidad de identidades epistemológicas entre sus profesores, que se manifiestan en la variedad de formas en que conciben la enseñanza de la psicología y se relacionan con la práctica educativa, por lo que no es extraño encontrar entre sus docentes pluralidad de formas de acercarse al conocimiento, a la enseñanza y a la evaluación (Covarrubias, 2003; Covarrubias \& Camarena, 2010).

Como estudio de caso elegimos una propuesta deconstructiva que nos permitiera interpretar las estructuras discursivas que producen los docentes para "entender" o comprender qué pasa en un contexto en particular, priorizando sus lógicas personales que informan sobre su práctica educativa (Stake, 1999), a partir de sus representaciones sociales y, en específico, las que atañen al enfoque por competencias, con la intención de introducirnos a lo que no es visible, a las estructuras subyacentes que nos permitieran explicar lo que aparentemente parece superficial y sin sentido, al tiempo que como investigadores nos comprometiera a ubicarnos en el lugar de los 
otros y observar los fenómenos sociales desde el punto de vista de ellos para someterlos al análisis y la interpretación.

Las representaciones sociales constituyen una oportunidad invaluable para acercarnos a la comprensión de los marcos de referencia que sustentan y fundamentan las identidades profesionales y pedagógicas de los profesores, y las formas en que las expresan o proyectan durante el acto educativo. Permiten conocer cómo obtienen, construyen o reconstruyen el propio conocimiento disciplinar y profesional del que participan, y los significados que atribuyen a las prácticas pedagógicas a las que se adscriben.

Consideramos que el conocer las posiciones que sobre el enfoque por competencias se tienen desde los directamente implicados en el proceso educativo permite aportar información para su problematización como innovación educativa. Permite, además, aportar conocimiento en este campo de investigación y, sobre todo, posibilita proponer formas y cambios para la adopción de mejores prácticas docentes; en tanto, y como apunta Senge (2002), si se quiere mejorar un sistema escolar, es necesario investigar primero el significado que atribuyen los docentes a las reformas e innovaciones para generar cambios reales en las instituciones educativas.

\section{Representaciones sociales en la educación.}

Como unidad de análisis, las representaciones en el ámbito educativo cobran gran significado ya que a partir de ellas se develan los fenómenos que no se aprecian a simple vista, pero que afectan o influyen en el proceso de enseñanza y aprendizaje.

Para Moscovici (1986), la representación social es una modalidad particular del conocimiento cuya función es la elaboración de los comportamientos y la comunicación entre los individuos. Es un corpus organizado de conocimientos y una de las actividades psíquicas gracias a las cuales los hombres hacen tangible la realidad física y social, se integran en un grupo o en una relación cotidiana de intercambios, liberan los poderes 
de su imaginación. Es un saber práctico de sentido común que permite el intercambio social, la transmisión y la difusión de ese saber "ingenuo".

Abric (2001) aclara que las representaciones sociales son, a la vez, producto y proceso de una actividad mental en la que el individuo o grupo reconstruye la realidad que enfrenta y le atribuye una significación específica. En este sentido, no son exclusivamente cognitivas; más bien, son construcciones sociocognitivas en tanto integran componentes individuales o psicológicos como sociales; es decir, suponen un sujeto activo cognitivamente de acuerdo con las reglas que rigen los procesos cognitivos, y la puesta en práctica de esos procesos cognitivos dependerá de las condiciones sociales en que las representaciones se construyen o se transmiten.

Las representaciones sociales conllevan un papel fundamental en las prácticas y en la dinámica de las relaciones sociales en tanto responden a necesidades y funciones esenciales: permiten entender y explicar la realidad (un saber práctico de sentido común), definen la identidad y salvaguardan la especificidad de los grupos (sitúan a los individuos y a los grupos en el campo social), orientan los comportamientos y las prácticas (constituyen una guía para la acción), permiten explicar y justificar las posturas y los comportamientos (justifican conductas, acciones realizadas) (Abric, 2011).

Su importancia en el campo educativo, y en particular en un espacio universitario como el que nos interesa, es que aportan información reveladora para ajustar acciones y prácticas educativas durante la formación profesional con base en las necesidades del profesorado y del estudiantado, de acuerdo con las condiciones institucionales y contextuales donde opera un currículo.

\section{Método}

El objetivo de la investigación que reportamos fue analizar las representaciones que docentes universitarios que participan en la enseñanza de la psicología en un campo universitario de la UNAM tienen sobre el enfoque de la Educación Basada en Competencias (EBC), y los sentidos que le otorgan 
para su aceptación o rechazo, si lo emplean en su práctica docente y, en su caso, el tipo de competencias que les interesa promover.

Por nuestro objeto de estudio e interés en un caso en particular, empleamos la metodología de corte cualitativo con enfoque descriptivo e interpretativo (Erickson, 1989), y elegimos el estudio de caso como proceso de indagación (Stake, 1999), al permitirnos obtener las representaciones de los docentes en su contexto educativo y desde una perspectiva psicosocial.

La investigación cualitativa o interpretativa se centra en el significado humano en la vida social y utiliza como criterio básico de validez los significados inmediatos y locales de las acciones, según se definen desde el punto de vista de los actores (Erickson, 1989). Esta metodología rescata la naturaleza socialmente construida de la realidad y la estrecha relación entre el investigador y la realidad investigada; por ello, la producción del conocimiento válido y relevante se concibe como un proceso de construcción de nuevos significados y representaciones a partir del contraste de las interpretaciones de los diferentes sujetos que participan de una misma realidad. En esta forma de comprender el comportamiento humano se pone énfasis en la hermenéutica como proceso de interpretación de los fenómenos humanos y en la subjetividad como dimensión de análisis primordial en la comprensión de la naturaleza humana (Pérez Gómez, 1998).

No obstante, esta metodología también implica un proceso activo, sistemático y riguroso de indagación dirigida, en la cual se toman decisiones sobre lo investigable en tanto se está en el campo de estudio, aunque sin la pretensión de alcanzar la generalidad de los datos, aun cuando estos puedan llegar a ser similares en contextos diferentes (Taylor \& Bogdan, 1990).

Como estudio de caso, nuestra responsabilidad se dirigió a la comprensión de las formas en que los profesores piensan su práctica docente, a lo que subyace, a lo que da sentido, lo que sin ser visible puede acercarnos a la comprensión de un quehacer docente dentro de una práctica educativa en particular, sin la intención de llegar a la generalización de los datos, ya que los fenómenos sociales y del comportamiento obedecen fundamentalmente a 
razones de carácter contextual, y en los que las historias biográficas, sociales y profesionales de sus actores le impregnan su particularidad, por lo que cada contexto tiene su carácter concreto e irrepetible y de su estudio no se extrae la comprobación de hipótesis de carácter general (Stake, 1999). En otras palabras, nuestra intención no fue llegar a premisas o leyes generales.

La consistencia y el rigor de los datos y las conclusiones provisionales lo intentamos alcanzar resaltando la identificación de diferentes puntos de vista y perspectivas o ángulos diferenciadores, en el contraste permanente de las indagaciones, las inferencias provisionales y las hipótesis de trabajo que se van decantando como fruto de la reflexión, del debate y del contraste mediante elaboraciones teóricas con los registros de la realidad. Los conocimientos teóricos, como herramientas conceptuales, adquieren su potencialidad dentro de un proceso discursivo de búsqueda e intervención en la realidad, como instrumentos para enriquecer y fundamentar la deliberación (Guba, 1983).

En tal sentido, no buscamos ajustar el conocimiento generado en la investigación a un marco teórico cerrado o verificar o formular una teoría sino, más bien, mantuvimos una perspectiva abierta para encontrar las pistas teóricas que pudieran dar respuesta o pudieran apoyar y fundamentar lo que íbamos extrayendo desde las primeras exploraciones de la investigación. Por lo tanto, tampoco establecimos categorías a priori o agrupamos los datos en categorías previamente predeterminadas por una teoría; por el contrario, estas fueron el resultado de un análisis minucioso, gradual y reflexivo para obtenerlas.

\section{Sobre el universo de estudio.}

De acuerdo con nuestro objeto de estudio, se invitó a profesores que fueran representativos de la planta docente de la carrera de Psicología de uno de los campos universitarios de la UNAM, ubicado al norte de la zona metropolitana de la Ciudad de México, y tuvieran disponibilidad para la entrevista. Se conformó así una muestra no probabilística, pero se intentó 
que fueran docentes que provinieran de las diferentes áreas que operan en la carrera, de diferentes turnos, sexo y antigüedad en la carrera.

Participaron 35 docentes, de los cuales 15 son varones y 20 mujeres: 22 del turno matutino, 3 del vespertino y 10 con turno mixto; con un rango muy amplio de antigüedad: de 2 meses a 39 años. Cada docente estaba adscrito a una de las nueve áreas académicas que contempla la carrera: Social Teórica, Social Aplicada, Métodos Cuantitativos, Psicología Clínica, Educación Especial y Rehabilitación, Desarrollo y Educación, Metodología, Experimental Humana y Experimental Animal (en la Tabla 2 se puede apreciar su distribución).

El número de docentes que participó se determinó con base en la saturación o reiteración de la información que se iba obteniendo (Saltalamacchia, 1992).

\section{Sobre la herramienta metodológica.}

Utilizamos la entrevista semiestructurada como fuente de información, ya que entre sus principales características están su flexibilidad y dinamismo para conseguir, de forma menos tensa y más casual, las representaciones que los docentes asignaran a la EBC (Taylor \& Bodgan, 1990).

Las entrevistas se aplicaron de manera individual y libre en los meses de octubre y diciembre de 2013, a partir de un guion de preguntas orientadoras o nucleares que aseguraran explorar las dimensiones básicas de la investigación. El guion contempló las siguientes preguntas: ¿Qué piensa usted acerca del debate que existe en cuanto al enfoque de la educación basada en competencias? ¿Utiliza en sus cursos este enfoque? ¿Por qué? ¿Promueve el desarrollo de competencias en sus clases? ¿Qué competencias le interesa promover en sus estudiantes? Las entrevistas fueron grabadas y transcritas para su análisis. 


\section{Sobre la construcción de las categorías teórico-analíticas.}

El proceso analítico-interpretativo de la información obtenida contempló la búsqueda de elementos comunes en todas las entrevistas y la relación de estos elementos con conceptos o categorías teóricas pertenecientes al campo de estudio sobre la enseñanza, lo que permitió la construcción de categorías teórico-analíticas (Hammersley \& Atkinson, 1994). En la Tabla 1 presentamos un ejemplo de la categorización construida.

Ello posibilitó organizar y presentar la información en torno a tres ejes principales: empleo /no empleo del enfoque por competencias, representaciones en contra y representaciones a favor del enfoque. 


\section{Tabla 1.}

Ejemplo de categorización de la información obtenida en las entrevistas aplicadas a los profesores(as).

\begin{tabular}{|c|c|c|c|c|}
\hline \multicolumn{5}{|c|}{$\begin{array}{l}\text { Pregunta } N^{\circ} 1 \\
¿ Q u e ́ \text { piensa usted acerca del debate que existe en cuanto al enfoque de la educación basada en competencias? }\end{array}$} \\
\hline Núm. Entrevista & Transcripción & $\begin{array}{c}\text { Categoría } \\
\text { social }\end{array}$ & $\begin{array}{l}\text { Categoría } \\
\text { analítica }\end{array}$ & $\begin{array}{l}\text { Categoría } \\
\text { teórica }\end{array}$ \\
\hline $\begin{array}{l}\text { (E12, Social Teórica, } \\
\text { p. } 1,3)\end{array}$ & $\begin{array}{l}\text { "No estoy de acuerdo } \\
\text { con el modelo, así } \\
\text { que está acabando con } \\
\text { la inteligencia de los } \\
\text { mexicanos, que a la larga } \\
\text { va a acabar con eso. [...] } \\
\text { la competencia forma un } \\
\text { pensamiento tecnócrata; } \\
\text { entonces no te permite } \\
\text { buscar relaciones más } \\
\text { profundas porque } \\
\text { implica pensar y, luego, } \\
\text { pensar te duele la cabeza } \\
\text {... me parece que no } \\
\text { sirve para formar gente } \\
\text { que haga propuestas, } \\
\text { que busque soluciones, } \\
\text { o sea, va a ser gente que } \\
\text { va a ser funcional en una } \\
\text { empresa, en un lugar, es } \\
\text { mano de obra barata, en } \\
\text { otras palabras" }\end{array}$ & $\begin{array}{l}\text { "está acabando } \\
\text { con la inteligencia } \\
\text { de los mexicanos; } \\
\text { forma un } \\
\text { pensamiento } \\
\text { tecnócrata; no } \\
\text { sirve para formar } \\
\text { gente que haga } \\
\text { propuestas, que } \\
\text { busque soluciones, } \\
\text { o sea, va a ser } \\
\text { gente que va a ser } \\
\text { funcional en una } \\
\text { empresa, en un } \\
\text { lugar, es mano de } \\
\text { obra barata" }\end{array}$ & $\begin{array}{l}\text { Formación } \\
\text { técnica, } \\
\text { tecnocrática, } \\
\text { funcional }\end{array}$ & $\begin{array}{l}\text { Orientación } \\
\text { tecnocrática y } \\
\text { funcional }\end{array}$ \\
\hline
\end{tabular}




\begin{tabular}{|c|c|c|c|c|}
\hline $\begin{array}{l}\text { (E7, Desarrollo y } \\
\text { Educación, p. 1) }\end{array}$ & $\begin{array}{l}\text { “... lo que a mí más } \\
\text { me preocupa del } \\
\text { modelo de enseñanza } \\
\text { por competencias es } \\
\text { que se enmarca en una } \\
\text { política educativa que se } \\
\text { orienta básicamente a la } \\
\text { formación técnica... está } \\
\text { poniendo al mercado } \\
\text { como quien define las } \\
\text { competencias en las } \\
\text { que se tiene que formar } \\
\text { a la persona para que } \\
\text { sean útiles a ese sistema } \\
\text { económico...” }\end{array}$ & $\begin{array}{l}\text { “se enmarca } \\
\text { en una política } \\
\text { educativa que } \\
\text { se orienta } \\
\text { básicamente a } \\
\text { la formación } \\
\text { técnica... está } \\
\text { poniendo al } \\
\text { mercado cómo } \\
\text { quien define las } \\
\text { competencias } \\
\text {... para que } \\
\text { sean útiles a } \\
\text { ese sistema } \\
\text { económico...” }\end{array}$ & $\begin{array}{l}\text { Formación } \\
\text { técnica, al } \\
\text { servicio del } \\
\text { mercado }\end{array}$ & $\begin{array}{l}\text { Orientación } \\
\text { tecnocrática y } \\
\text { funcional }\end{array}$ \\
\hline $\begin{array}{l}\text { (E2, Métodos } \\
\text { Cuantitativos, p. 1) }\end{array}$ & $\begin{array}{l}\text { “... la ventaja más } \\
\text { importante del modelo } \\
\text { de competencias, a } \\
\text { diferencia del modelo } \\
\text { tradicional, marcaba } \\
\text { la necesidad de que } \\
\text { no fuera a nivel de } \\
\text { repetición como lo hacía } \\
\text { el sistema tradicional; } \\
\text { sino más bien que ya } \\
\text { fuera de ejecución... } \\
\text { pretende que se } \\
\text { muestren las habilidades } \\
\text { necesarias para } \\
\text { mostrar que realmente } \\
\text { manejas o dominas el } \\
\text { conocimiento" }\end{array}$ & $\begin{array}{l}\text { "la necesidad } \\
\text { de que no } \\
\text { fuera a nivel de } \\
\text { repetición como } \\
\text { lo hacía el sistema } \\
\text { tradicional; sino } \\
\text { más bien que ya } \\
\text { fuera de ejecución } \\
\text {... mostrar que } \\
\text { realmente manejas } \\
\text { o dominas el } \\
\text { conocimiento" }\end{array}$ & $\begin{array}{l}\text { Sentido } \\
\text { práctico, de } \\
\text { ejecución }\end{array}$ & $\begin{array}{l}\text { Orientación } \\
\text { funcional, } \\
\text { laboral y } \\
\text { conductual }\end{array}$ \\
\hline
\end{tabular}

\section{Resultados}

Por cuestiones de espacio, presentamos únicamente las tendencias más representativas de las indagaciones realizadas en los tres ejes conformados para su análisis, apoyándonos solo en algunos de los argumentos de los profesores para sustentar las interpretaciones realizadas. 


\section{Empleo/no empleo de la educación basada en competencias.}

En la Tabla 2 se puede observar que la tendencia en las posturas de los profesores con respecto a la implementación del enfoque por competencias en su práctica educativa no es a favor de su empleo, en tanto el 65.7\% (23 docentes) lo rechazan y el 34.3\% (12 docentes) lo aceptan. Sin embargo, encontramos que la mayoría de los profesores del área de Psicología Social Aplicada lo aceptan y emplean, mientras que ninguno de los profesores del área de Psicología Social Teórica y de Psicología Clínica lo aceptan.

Es decir, en general parece existir preferencia o rechazo del enfoque en algunas áreas académicas de la carrera, mientras que, en otras, las posturas se encuentran prácticamente divididas.

\section{Tabla 2.}

Distribución de profesores(as) que aceptan o no aceptan el modelo por competencias por área de adscripción, sexo, turno y antigüedad.

\begin{tabular}{|c|c|c|c|c|c|c|c|c|}
\hline \multirow[b]{2}{*}{$\begin{array}{l}\text { Área de } \\
\text { adscripción }\end{array}$} & \multirow{2}{*}{$\begin{array}{l}\text { Núm. de } \\
\text { profesores } \\
\text { que SÍ } \\
\text { aceptan/ } \\
\text { emplean la } \\
\text { EBC }\end{array}$} & \multirow{2}{*}{$\begin{array}{l}\text { Núm. de } \\
\text { profesores que } \\
\text { NO aceptan/ } \\
\text { emplean la } \\
\text { EBC }\end{array}$} & \multicolumn{2}{|c|}{ Sexo } & \multicolumn{3}{|c|}{ Turno } & \multirow[b]{2}{*}{ Antigüedad } \\
\hline & & & $\mathbf{M}$ & $\mathbf{F}$ & Mat. & Vesp. & Mix & \\
\hline $\begin{array}{l}\text { Social } \\
\text { Aplicada }\end{array}$ & $\underline{4}$ & 1 & 2 & 3 & 3 & 1 & 1 & 13-35 años \\
\hline Social Teórica & & $\underline{6}$ & 2 & 4 & 5 & & 1 & 2 meses-35 años \\
\hline $\begin{array}{l}\text { Métodos } \\
\text { Cuantitativos }\end{array}$ & 1 & 2 & 2 & 1 & 3 & & & 21-33 años \\
\hline $\begin{array}{l}\text { Psicología } \\
\text { Clínica }\end{array}$ & & $\underline{4}$ & & 4 & 1 & & 3 & 2 -21 años \\
\hline Educación & & & & & & & & \\
\hline $\begin{array}{l}\text { Especial y } \\
\text { Rehabilitación }\end{array}$ & 2 & 2 & 2 & 2 & 2 & & 2 & 4-28 años \\
\hline $\begin{array}{l}\text { Desarrollo y } \\
\text { Educación }\end{array}$ & 1 & 2 & 2 & 1 & 2 & 1 & & 6 meses-39 años \\
\hline Metodología & 1 & 1 & & 2 & 1 & 1 & & 20-30 años \\
\hline
\end{tabular}




\begin{tabular}{|c|c|c|c|c|c|c|c|c|}
\hline $\begin{array}{l}\text { Experimental } \\
\text { Humana }\end{array}$ & 2 & 3 & 2 & 3 & 4 & & 1 & 28-32 años \\
\hline $\begin{array}{l}\text { Experimental } \\
\text { Animal }\end{array}$ & 1 & 2 & 1 & 2 & 1 & & 2 & 9-30 años \\
\hline $\begin{array}{l}\text { TOTALES } \\
\mathbf{N}=\mathbf{3 5}\end{array}$ & $\begin{array}{c}12 \\
(34.3 \%)\end{array}$ & $\begin{array}{c}23 \\
(65.7 \%)\end{array}$ & 15 & 20 & 22 & 3 & 10 & $\begin{array}{l}2 \text { meses-39 } \\
\text { años }\end{array}$ \\
\hline
\end{tabular}

Pero lo importante aquí es el tipo de representaciones y sentidos que los profesores le otorgan al enfoque para su aceptación o rechazo. Al parecer, el tipo de contenidos y saberes profesionales que se contemplan en las áreas y asignaturas en las que participan los docentes median sus representaciones sobre el enfoque. Por ejemplo, a los profesores que aceptan el enfoque del área de Social Aplicada les resulta de utilidad para promover experiencias prácticas que sirvan a los estudiantes para su posterior ejercicio profesional:

este modelo es importante para mí si el alumno adquiera eso que sea competencia o habilidad, pero que pueda adquirirlo en este caso conmigo en la práctica ... A veces uno como profesor, en la práctica, uno lo lleva a cabo y al alumno le ofrece estabilidad [...] los alumnos, por lo menos con los que yo platico, ellos me dicen que están trabajando en los procesos de intervención en los lugares donde estaban y adquirieron conmigo mucha experiencia, porque aprendieron cosas como trabajar con grupos o poder organizar el trabajo con niños de primaria, que ha sido difícil pero que al final lo logran... (E3, Social Aplicada, p. 2).

Otros valoran el desarrollo de actitudes que promueve el enfoque:

Yo soy psicólogo humanista, trabajo mucho lo que son las competencias actitudinales, el ser empático, el ser asertivo, el tener una ética profesional (E1, Social Aplicada, p. 1).

Por el contrario, para los profesores del área de Social Teórica que rechazan el enfoque, su problema estriba en que este "solo forma técnicos... es una idea muy clara de acabar con las humanidades" en lugar de "formar 
pensadores, hacer científicos" que les sirvan para alcanzar niveles de eficiencia:

No estoy de acuerdo con el modelo, así que está acabando con la inteligencia de los mexicanos, que a la larga va a acabar con eso. [...] la competencia forma un pensamiento tecnócrata; entonces no te permite buscar relaciones más profundas porque implica pensar $\mathrm{y}$, luego, pensar te duele la cabeza... me parece que no sirve para formar gente que haga propuestas, que busque soluciones, o sea, va a ser gente que va a ser funcional en una empresa, en un lugar, es mano de obra barata, en otras palabras (E12, Social Teórica, p. 1,3).

Sin embargo, el pensar, el análisis, la crítica y la autorreflexión se convierten en una aspiración común a promover entre los estudiantes tanto para profesores que enseñan contenidos prácticos como teóricos, como es el caso de docentes de psicología clínica que no aprueban o no emplean el enfoque:

No lo empleo, no directamente, no de manera establecida [...] Me interesa que puedan poner en tela de juicio los conceptos, que puedan desarrollar como un análisis crítico y que ese análisis pueda tener un sustento sólido y consolidado, que puedan hacer una buena crítica. Eso y la parte del auto-análisis... que las desarrollen para su propia vida. Eso es algo que yo promuevo. Psicología que no sirve para tu propia vida me parece psicología tonta (E25, Psicología Clínica, pp. $1,2)$.

Lo cierto es que, con todo y los diferentes tipos de contenidos y saberes que cada área de especialización de la psicología contempla como propios, como señala Coll (2007), la adquisición o desarrollo de una competencia, por su naturaleza, está firmemente relacionada con la adquisición o movilización de una serie de recursos - conocimientos, habilidades, actitudes- que variarán de acuerdo con cada disciplina, área, programa o asignatura y con su perfil de formación profesional. Sin embargo, para la psicología como ciencia social, la intencionalidad desempeña un papel importante en el estudio de la acción y el pensamiento humanos, por lo que la valoración 
de las competencias actitudinales adquiere importancia en cualquiera de las áreas de especialización de la psicología.

\section{Representaciones en contra del enfoque por competencias.}

Si bien encontramos posturas tanto a favor como en contra del enfoque por competencias entre los profesores entrevistados, la balanza se inclina hacia los que están en contra, y aunque son diversas sus representaciones para su rechazo, las mayormente enunciadas tienen que ver con su sentido empresarial y técnico, así como con las dificultades para llevarlo a la práctica.

\section{Su orientación globalizadora y tecnocrática.}

En general, los argumentos expuestos por los docentes que tienen una clara postura en contra de la implementación del enfoque por competencias giran en torno a que este responde a las necesidades derivadas de la globalización que, vinculadas a las políticas neoliberales y al mundo empresarial, hacen que los profesores lo pongan bajo sospecha:

Estoy en contra, parece que, ante las condiciones políticas que tenemos en México, lo que se necesita es gente que no piense, gente que tenga condiciones de mucha tecnología, como si fueran técnicos; lo que se ha hecho es cambiar el sistema educativo en función de las necesidades actuales derivadas de la globalización (E8, Social Aplicada, p. 1).

... lo que a mí más me preocupa del modelo de enseñanza por competencias es que se enmarca en una política educativa que se orienta básicamente a la formación técnica... está poniendo al mercado como quien define las competencias en las que se tiene que formar a la persona para que sean útiles a ese sistema económico... (E7, Desarrollo y Educación, p. 1).

Argumentos que coinciden con los puntos más controvertidos que se han expuesto en las publicaciones al respecto. Como se ha documentado por los especialistas, el tema de las competencias en el ámbito educativo proviene 
del mundo del trabajo, en el que se adoptan como respuesta estratégica para elevar la competitividad de las empresas en el contexto de una economía globalizada que exige nuevos perfiles profesionales para hacer frente a las presiones económicas y sociales de los procesos mundiales enmarcados en una sociedad del conocimiento bajo la tutela de las tecnologías de la información y la comunicación (Climent, 2009; Díaz-Barriga, 2011; PlanasColl, 2013).

\section{Sus dificultades de aplicación.}

Para otros profesores que no aprueban el enfoque por competencias o no lo emplean, el problema radica en la dificultad o complejidad que implica llevarlo a la práctica en las aulas.

Para algunos, la dificultad estriba en las resistencias que genera cualquier innovación educativa:

vivimos inmersos en un contexto donde aún no nos separamos de los enfoques tradicionales en educación, no creo que sea muy viable que se pueda aplicar [...] se tendría que cambiar la estructura formativa de los docentes de fondo, o sea, desde el principio, desde las bases y desde la manera en como ellos se preparan para ser docentes, tendrían que estar capacitados y tendrían que estar sensibilizados para entender este nuevo modo de enseñar (E1, Social Aplicada, pp. 1-2).

Para otros, el desconocimiento del enfoque o sus bases conductistas es lo que obstaculiza ponerlo en práctica:

Yo creo que no se ha entendido muy bien el modelo de competencias, que muy poca gente lo conoce a cabalidad, debaten desde la ignorancia ¿no? Y, bueno, yo creo que este debate también tiene que ver con que este modelo de competencias es muy conductista, entonces a mucha gente no le gusta (E9, Experimental Humana, p. 1). 
Para otros, el problema reside en los estudiantes al no llegar a la universidad con las competencias previas que debieron desarrollar desde niveles escolares previos:

Estoy a favor, pero siempre y cuando se aplique al principio de los años escolares, que hubiera una generación donde se aplicara primero ahí, soy de la idea de que a estas alturas es muy difícil moldear competencias para la adquisición de aprendizaje en la licenciatura, ahorita aplicarlo no está resultando (E11, Experimental Humana, pp. 1-2).

Inclusive hay quien argumenta que no lo emplea porque no está aprobado por la institución: "No lo uso porque yo me rijo con el programa que está vigente en el H. Consejo Técnico" (E5, Metodología, p. 1).

A todas luces, las problemáticas que señalan los profesores son un reflejo del reto que al enfoque de las competencias se le ha imputado. Por un lado están las resistencias al cambio que, como en toda innovación educativa, es una condición muy conocida en tanto trastoca aspectos laborales, tareas, actividades e intereses de los docentes; y por otro se requiere clarificar su propia propuesta, lo cual significa construir un lenguaje que contenga tanto su propuesta como sus límites. Al respecto, Díaz Barriga (2006) en un artículo en el que analiza si el enfoque por competencias realmente representa una innovación o sólo es un disfraz de cambio, revisa los fundamentos conceptuales del término competencia así como algunas experiencias que se han establecido a partir del mismo en algunos ámbitos y niveles educativos en México. Concluye que existe ausencia de una reflexión conceptual que acompañe al enfoque por competencias como innovación educativa o, por lo menos, un dominio conceptual y técnico del significado del mismo, necesario para generar las adaptaciones que reclama cada realidad educativa. En general, observa que las acciones que demanda el enfoque no alcanzan a realizarse con cierta solidez dado que, como toda innovación en el sistema de educación en México, responde más a una declaración verbal que a una acción realizada por los docentes a partir del convencimiento de la importancia de 
este o, por lo menos, de un dominio conceptual y técnico del mismo que se materialice en la aplicación del enfoque, por lo que advierte: Si una certeza hay en este momento, es que no existe claridad en cómo tal enfoque se puede aplicar en la educación básica o en la educación superior (p. 17).

Esto es, se requiere evitar la diversidad tan amplia de interpretaciones que desde la perspectiva de las competencias se están elaborando en el campo de la educación. Además de obtener consensos sobre su conceptualización y entendimiento, se necesita de orientaciones precisas y formulaciones concretas para la definición, el diseño, organización e implementación de un plan o programa de estudios.

\section{Representaciones a favor del enfoque por competencias.}

Si bien son menos los profesores que aprueban la enseñanza por competencias, también son diversas las razones y significados que le atribuyen para su empleo. Con todo, entre los argumentos más representativos para su utilización se encuentran: los saberes técnicos y prácticos que el enfoque promueve y su adecuación a las necesidades laborales o del mercado de trabajo; pero también algunos rescatan la visión más sistémica y constructivista del enfoque al mencionar su función más integradora de saberes conceptuales, procedimentales y actitudinales que contempla.

\section{Su orientación funcional, laboral y conductual.}

Contraria y paradójicamente a las concepciones de los profesores que no aprueban la enseñanza por competencias debido a su utilidad práctica y su relación con las demandas del campo del trabajo, para otros docentes son estas características las que los llevan a tener una clara postura a favor de su implementación.

Al parecer, se adscriben al enfoque por competencias desde una perspectiva funcional ${ }^{2}$, laboral y conductual que enfatiza la necesidad de

2 El modelo funcional tiene su base en la escuela de pensamiento funcionalista, acogido por Inglaterra 
traducir las competencias en evidencias objetivas, "el saber hacer y el saber ser", en la que se descartan los conocimientos memorísticos y se prioriza la "ejecución" bajo criterios y evidencias de desempeño, enfatizando así un saber práctico efectivo:

la ventaja más importante del modelo de competencias, a diferencia del modelo tradicional, marcaba la necesidad de que no fuera a nivel de repetición como lo hacía el sistema tradicional; sino más bien que ya fuera de ejecución... pretende que se muestren las habilidades necesarias para mostrar que realmente manejas o dominas el conocimiento (E2, Métodos Cuantitativos, p. 1).

yo creo que sí es funcional porque antes trabajábamos mucho en la cuestión memorística y apuntes y redacción, cuestiones muy vacías ¿no?, era un trabajo muy plano [...] el concepto de competencias tiene que ver con que adquieran ciertas habilidades que les permitan a ellos mismos la autonomía de investigación y puedan darles solución a las problemáticas, yo no lo hago a un lado, hay que rescatar lo que es funcional (E27, Desarrollo y Educación, p. 1).

Se mira así a la EBC como aquella que permite solucionar problemas persistentes de nuestro sistema educativo, como es la enseñanza de tipo enciclopédico o memorística, que solo tiene aplicación en la escuela pero no en la realidad cotidiana de los estudiantes, y en su lugar se orienta a la resolución de problemas de su entorno mediante el desarrollo de competencias que los nuevos escenarios sociales exigen (Díaz Barriga, 2011).

Argumentos que acaban siendo precisamente entre los más extendidos para justificar el empleo del enfoque por competencias en la educación nacional, ligados a otro más extendido, que es el "educar para la vida" (Vaca, Aguilar, Gutiérrez, Cano \& Bustamante, 2015).

a través del sistema nacional de competencias nacionales NVQ. El enfoque enfatiza que todo lo que se aprendedebetenerunautilidadinmediataenlavida.LohaimpulsadolaOrganizaciónparalaCooperación y el Desarrollo Económico (OCDE) a través del programa de evaluación conocido como PISA (A. Díaz Barriga, 2011). 
Esta perspectiva de la EBC es promovida por el Proyecto Definición y Selección de las Competencias (DeSeCo, 2005) de la Organización para la Cooperación y el Desarrollo Económico (OCDE), que está orientado a responder a las presiones de la economía del mercado y a la preocupación internacional por la reforma de los sistemas educativos vigentes que son insatisfactorios. Parte de una concepción de corte conductual/laboral que pone atención, como advierte Moreno (2009), “a los resultados obtenidos por el individuo mediante una acción, decisión o forma de comportarse con respecto a las demandas que se le plantean, relacionadas, por ejemplo, con una posición profesional concreta, un papel social o un proyecto personal determinados (p. 72)":

\footnotetext{
el alumno se hace competente en el mercado al que ingrese, entonces tiene que saber de todo lo que se tiene que enfrentar allá afuera, si, y realmente no solo sirve que me digan que sí lo sabe hacer, sino que realmente lo tiene que ejecutar (E14, Educación Especial y Rehabilitación, p. 2).
}

Sin embargo, y como apuntábamos, el significado y uso que se dé al enfoque por competencias dependerá de la escuela de pensamiento con que se adopte una propuesta curricular por competencias, y en nuestro caso, de la óptica con la que lo miren los profesores.

\section{Su orientación integradora y constructivista en la formación de los estudiantes.}

Aunque son pocos, encontramos profesores que valoran el enfoque por competencias desde una concepción más sistémica y constructivista de la educación, cuya función es promover una formación más integral de los estudiantes que les permita una comprensión y explicación de los fenómenos, y no necesariamente los prepare, juzgue o evalúe a partir de la empleabilidad directa de lo aprendido; es decir, son profesores que se distancian del enfoque por competencias que promueve la formación exclusivamente "para el trabajo": 
porque les permite reflexionar, sintetizar, realizar, ser creativos, desempeñar o, más bien, promover actitudes que impliquen valores que les permitan desenvolverse en la sociedad de una manera más adecuada (E17, Social Aplicada, p. 2).

La perspectiva constructivista del enfoque por competencias, que no procede de las exigencias del mercado, sino más bien de sus repercusiones en la educación y como reflejo de la era global en los sistemas educativos, tiene sus orígenes en los planteamientos piagetianos y vigotskyanos, lo cual ha permitido generar una visión diferente del trabajo por competencias en la educación al volver a situar, por algunos de sus autores, la importancia de los enfoques centrados en el aprendizaje y de la labor de la escuela y del docente en disponer o crear espacios que permitan al estudiante, a partir de su acercamiento a objetos cognitivos, ir construyendo su propio andamiaje de información y construcción del conocimiento (Díaz Barriga, 2011). El argumento de otra profesora es muy consistente en este sentido:

Pues yo promuevo mucho la reflexión, o sea, siempre empiezo a discutir los casos de manera individual, les doy la asesoría así como en tutorías, pero ya cuando están atendiendo los casos les pregunto sobre los antecedentes del niño, la familia, la problemática, y entonces les voy dando como bombardeo de ideas de las que me vienen y el chico vaya analizando [...] si el estudiante se desvincula con la realidad que es cambiante y dinámica, entonces sí podría haber algunas deficiencias o contratiempos (E6, Educación Especial y Rehabilitación, pp. 3, 1).

Desde esta perspectiva, el contexto de aprendizaje cobra importancia donde no solo se requiere integrar los saberes construidos a nuevas situaciones, sino que las nuevas exigencias de la realidad operan como contexto de nuevos aprendizajes. Si bien esta perspectiva no es nueva en la educación, pues responde a la misma lógica de construcción del conocimiento de las propuestas de aprendizaje por proyectos, por problemas, por casos o por el ahora recurrido aprendizaje situado, cuyas bases se remontan al pensamiento de Dewey y, posteriormente, a los distintos planteamientos de la psicología genética y la teoría sociocultural, coloca al enfoque de la 
educación por competencias con un perfil muy distante a su perspectiva funcional, conductual y laboral.

\section{Discusión}

Como estudio de caso, no hemos pretendido que los resultados de esta investigación sean concluyentes ni exhaustivos. Nuestra intención, más bien, ha sido indagar si el enfoque por competencias representaría, para profesores de psicología de un espacio universitario en particular, una alternativa para resolver los problemas que se atribuyen a su enseñanza. En este sentido, los hallazgos encontrados son solo contextuales y locales; sin embargo, pretenden contribuir al conocimiento y al debate o controversias que ha generado su adopción en prácticamente todos los niveles educativos en México.

La noción de representaciones sociales como concepto y unidad de análisis fue esencial para aproximarnos a las formas en que los profesores piensan el enfoque por competencias y los sentidos o significados que le atribuyen, y al tener una proyección práctica, en tanto no son simples actitudes ante los hechos, sino que tienen ese componente activo que se proyecta en la acción, nos permitieron adentrarnos en las formas en que median su práctica pedagógica. En otras palabas, las representaciones no solo sirven como instrumento para el conocimiento de la realidad y su interpretación, sino también como un sistema para la planeación y el control, por lo que tienen que ver con las acciones o actividades para interactuar con el medio e influyen, como señalan Tabachnick y Zeichner (1982, citado por Gimeno, 1995), en las formas en que los profesores piensan su trabajo y en la forma en que dan significado a esas creencias a través de sus actos en clase (p. 2).

El sexo, la antigüedad en la docencia y el turno en el que desempeñan la labor docente no parecen estar relacionados con la aceptación o rechazo del enfoque por competencias entre los profesores participantes en la investigación; y aunque hallamos relación de algunas áreas académicas de la carrera con su aprobación o no aprobación, no la encontramos como una condición que se relacione con todas las áreas. 
Si bien la diversidad de representaciones y conceptualizaciones con respecto al enfoque por competencias que se decanta en la literatura y debate sobre el tema también se manifestó en esta investigación, en términos generales, la tendencia en los profesores entrevistados es el rechazo o no aprobación del enfoque. La mayoría argumenta que es un modelo funcionalista, conductual y empresarial, que justo son las críticas y cuestionamientos que se exponen en los debates que sobre el tema hacen los especialistas en currículo. Estas representaciones y críticas parecen proceder de la asociación o vinculación que se hace del enfoque por competencias con las políticas de corte neoliberal que subordinan la educación a las demandas del mercado laboral; concepción que parece proceder del origen del término competencias precisamente del mundo del trabajo y se traslada a la educación sin que necesariamente se compartan o coincidan las visiones que se tienen en los dos ámbitos.

Sin embargo, y más allá de la asociación del término al campo del trabajo, esta perspectiva ofrecería más posibilidades de articulación entre la educación y el mercado de trabajo, lo que disminuiría o resolvería el problema del desfase entre la formación profesional del psicólogo y las necesidades ocupacionales y sociales de nuestra sociedad, en tanto enfatiza la importancia del desempeño de los profesionistas en el ámbito del trabajo, se prioriza el "saber hacer y el saber ser" en aras de disminuir la formación de tipo enciclopedista, problema del que se acusa a la enseñanza de la psicología. No obstante, son menos los profesores que coinciden con esta perspectiva al plantear precisamente la necesidad de la articulación educación-empleo en la formación profesional del psicólogo.

Como podemos apreciar, al parecer no solo no hay consenso en la forma en que se representa o concibe el enfoque por competencias, sino tampoco en la visualización de la psicología como profesión entre los docentes. Si bien son menos, también hay profesores que se inclinan por el enfoque desde una perspectiva constructivista, docentes que están más preocupados por la formación integral de los estudiantes que por acortar 
la distancia entre la formación profesional y las necesidades del mercado laboral. Condición que confirma no solo las diversas maneras de representar el enfoque por competencias, sino también la formación profesional, lo que dificulta tener una perspectiva unificada en cuanto a los fines o propósitos de la educación superior. Esta heterogeneidad con la que se significa y se concibe la formación profesional y las innovaciones curriculares y educativas es tema por demás recurrente en la investigación educativa, como recurrentes son las implicaciones que esto conlleva para el éxito de las innovaciones pedagógicas.

\section{Referencias}

Abric, J. C. (2001). Las representaciones sociales: aspectos teóricos. En J. C. Abric (Dir.), Prácticas sociales y representaciones (pp. 11-32). México: Coyoacán.

Almeida, E., Guarneros, N., Limón, A., \& Román S. (1989). Presente y futuro del trabajo profesional del psicólogo en México. En J. Urbina (Comp.), El psicólogo: formación, ejercicio profesional y prospectiva (pp. 787-791). México: UNAM.

Castañeda, S. (1999). Algunos referentes para un análisis crítico para la preparación profesional del Licenciado en Psicología en México. Una experiencia colegiada. En G. Vázquez y J. Martínez (Eds.), La formación del Psicólogo en México (pp. 25-41). México: Universidad Latinoamericana.

Castaño, D. (1989). Prospectiva del ejercicio profesional de la psicología en México. En J. Urbina (Comp.), El psicólogo: formación, ejercicio profesional y prospectiva (pp. 779-785). México: UNAM.

Climent, J. B. (2009). Reflexiones sobre la Educación Basada en Competencias. Revista Complutense de Educación, 21(1), 91-106.

Coll, C. (2007). Las competencias en la educación escolar: algo más que una moda y mucho menos que un remedio. Aula de innovación educativa, 161, 34-39. 
Covarrubias, P. (2003). Currículum, disciplina y profesión, desde la perspectiva de los académicos de Psicología Iztacala (Tesis de doctorado). Universidad Nacional Autónoma de México, México D.F.

Covarrubias, P., \& Camarena, O. (2010). Construcción del conocimiento e identidad profesional. México: UNAM; Castellanos Editores.

Covarrubias, P., \& Casarini, M. (2013). Los actores del currículo en México: Un campo de conocimiento en constitución. En A. Díaz Barriga (Coord.), La investigación curricular en México. La primera década del siglo XXI (2002-2012) (pp. 197-262). México D.F.: Colección Estados del Conocimiento: COMIE-ANUIES.

DeSeCo, O. E. C. D. (2005). The definition and selection of key competencies. Executive summary. OCDE-USAID. Recuperado de http://www. deseco. admin. ch/bfs/deseco/en/index/02. html.

Díaz Barriga, Á. (2006). El enfoque de competencias en la educación: ¿Una alternativa o un disfraz de cambio? Perfiles educativos, 28(111), 7-36.

Díaz Barriga, Á. (2011). Competencias en educación. Corrientes de pensamiento e implicaciones para el currículo y el trabajo en el aula. Revista Iberoamericana de Educación Superior, 2(5), 3-24.

Díaz Barriga, A. (2013). Secuencias didácticas. ¿Un problema del enfoque de competencias o un reencuentro con perspectivas didácticas? Profesorado. Revista del currículum y formación del profesorado, 17(3), 11-33. Recuperado de http://www.ugr.es/local/recfpro/rev173ART1.pdf

Díaz Barriga, F. et al. (2013). Innovaciones curriculares. En A. Díaz Barriga (Coord.). La investigación curricular en México. La primera década del siglo XXI (2002-2012) (pp. 109-196). México D.F.: Colección Estados del Conocimiento: COMIE-ANUIES.

Erickson, F. (1989). Métodos cualitativos de investigación sobre la enseñanza. En M. C. Wittrock, La investigación de la enseñanza, 2. Barcelona: Paidós.

Gimeno, S. (1995). El currículum: una reflexión sobre la práctica (5 $\left.5^{\mathrm{a}} \mathrm{ed}.\right)$. Madrid: Morata.

Gimeno, S., \& Pérez Gómez, A. (1989). (Comp.). La enseñanza, su teoría y su práctica. Madrid: Akal-Universitaria. 
Guba, E. (1983). Criterios de credibilidad en la investigación naturalista. En Gimeno, S. \& Pérez Gómez, A. (Comp.) (1989). La enseñanza, su teoría y su práctica (pp.148-165). Madrid: Akal-Universitaria.

Hammersley, M., \& Atkinson, P. (1994). Etnografía. Métodos de Investigación. México: Paidós.

Harrsch, C. (1998). La identidad del psicólogo (2ª reimp.). México: Pearson Education.

Herrera, A. (1993). La formación profesional del psicólogo. Perfiles Educativos, 59, pp. 31-44. Recuperado de http://www.iisue.unam.mx/ perfiles/perfiles_articulo.php?clave=1993-59-31-44

Lara, L. (1989). La profesión de psicólogo: un análisis de sus antecedentes, situación actual y futura. En J. Urbina (Comp.), El psicólogo: formación, ejercicio profesional y prospectiva (pp. 769-778). México: UNAM.

Moreno T. (2009). Competencias en educación superior: un alto en el camino para revisar la ruta de viaje. Perfiles educativos, 31(124), 69-92.

Moreno, T. (2010). El currículum por competencias en la universidad: más ruido que nueces. Revista de Educación Superior, 39(154), 77-90. Recuperado de http://www.redalyc.org/pdf/604/60418903004.pdf

Moscovici, S. (1986). Pensamiento y vida social. Psicología social y problemas sociales. Psicología social II. Barcelona: Paidós.

Pérez Gómez, A. (1998). La cultura escolar en la sociedad neoliberal. Madrid: Morata.

Planas-Coll, J. (2013). El contrasentido de la enseñanza basada en competencias. Revista Iberoamericana de Educación Superior, 4(10), 75-92. http://dx.doi.org/10.1016/S2007-2872(13)71925-5

Preciado, H., \& Rojas, L. A. (1989). Notas sobre la enseñanza de la psicología en México: estado actual y perspectivas de desarrollo. Revista de la Educación Superior, 18(72), 57-72.

Saltalamacchia, H. (1992). Historia de vida. Puerto Rico: CIJUP.

Senge, P. (2002). Escuelas que aprenden. Bogotá: Norma.

Stake, R. E. (1999). Investigación con estudio de casos. Madrid: Morata. 
Taylor, S. J., \& Bogdan, R. (1990). Introducción a los métodos cualitativos de investigación. Barcelona: Paidós.

Vaca, J., Aguilar, V., Gutiérrez, F., Cano, A., \& Bustamante, J. A. (2015). ¿Qué demonios son las competencias? Aportaciones del constructivismo clásico y contemporáneo. México: Universidad Veracruzana; Instituto de Investigaciones de la Educación. Recuperado de http://www. uv.mx/psicologia/files/2015/07/Vaca-Uribe-J.-Que-demonios-son-lascompetencias.pdf

Victorino, L., \& Medina, G. (2007). Educación basada en competencias y el proyecto Tuning en Europa y Latinoamérica. Ide@s, 3(39), 97-114.

Winterton, J., Delamare, F., \& Stringfelow, E. (2006). Typology of knowledge, skills and competences: clarification of the concept and prototype. Luxemburgo: CEDEFOP Reference series 64, Office for Official Publications of the European Communities. Recuperado de http://www. cedefop.europa.eu/en/publications-and-resources/publications/3048

Young, R. E. (1981). A study of teacher epistemologies. Australian Journal of Education, 25(2), 194-208. http://dx.doi. org/10.1177/000494418102500208

Zanatta, E., \& Yurén, T. (2012). La formación profesional del psicólogo en México: trayecto de la construcción de su identidad disciplinar. Enseñanza e Investigación en Psicología, 17(1), 151-170. Recuperado de http://www.cneip.org/documentos/10.pdf 